\title{
Diet supplementation with commercial enzymatically-hydrolyzed egg white peptides ameliorates the severity of allergy in mouse model of egg white allergy
}

\author{
Akihiro Maeta, Risako Katahira, Matsushima Marin, Kyoko Takahashi
}

\begin{abstract}
Background: Commercial spray-dried egg white (S-EW) is often used for oral immunotherapy (OIT). Peptifine ${ }^{\varpi}$ (PF), a commercial preparation of enzymatically-hydrolyzed egg white (EW) peptides, is considered safe OIT food.

Objective: Here, we examined the therapeutic effect of PF or S-EW in a mouse model of EW allergy.

Methods: Sensitized female BALB/c mice underwent 4-week OIT by supplementation of $19 \%$ casein diet with $1 \%$ PF (PF group) or S-EW (S-EW group), and non-supplementation of $20 \%$ casein diet (non-OIT group). Non-sensitized mice acted as the non-allergy group. Oral and intraperitoneal EW challenges were performed and allergic biomarkers were determined.

Results: Changes in rectal temperature after oral challenge were comparable in the allergy groups. However, after intraperitoneal challenge, rectal temperature decrease in the PF and S-EW groups was lower than in the non-OIT group. After 4 weeks, plasma levels of ovalbumin- and ovomucoid-specific antibodies were assessed; IgE levels in the $\mathrm{PF}$ and S-EW groups were lower than those in the non-OIT group. Interleukin-4 secretion after EW challenge was significantly lower in splenocyte cultures derived from PF and S-EW groups than in the non-OIT group. Furthermore, the percentage of $\mathrm{CD}^{+}$Foxp $^{+}$splenocytes in the PF group was significantly higher than that in the non-OIT and S-EW groups. Oral gavage of $40 \mathrm{mg} \mathrm{PF}$ did not induce an allergic response in sensitized mice with EW.
\end{abstract}

Conclusion: Diet supplementation with 1\% PF mildly ameliorated the severity of allergy in mouse with EW allergy, indicating that PF is a safe OIT food.

Key words: hen's egg allergy, enzymatically-hydrolyzed egg white peptide (Peptifine ${ }^{\varpi}$ ), egg white-specific allergic mouse model, spray-dried egg white (S-EW), oral immunotherapy (OIT)

\section{Citation:}

Maeta, A., Katahira, R., Marin, M., Takahashi, K. (2022).

Diet supplementation with commercial enzymatically-hydrolyzed egg white peptides ameliorates the severity of allergy in a mouse model of egg white allergy. Asian Pac J Allergy Immunol, 40(4), 359-367. https://doi.org/10.12932/ap-080219-0489

\section{Affiliation:}

Department of Food Science and Nutrition, School of Human Environmental Sciences, Mukogawa Women's University, Hyogo, Japan

\section{Corresponding author:}

Kyoko Takahashi

Department of Food Science and Nutrition, School of Human

Environmental Sciences, Mukogawa Women's University,

6-46, Ikebiraki-cho, Nishinomiya, Hyogo, 663-8558, Japan

E-mail: taka@mukogawa-u.ac.jp

\section{Introduction}

In Japan, hen's egg allergy is the most common food hypersensitivity identified in the pediatric population. ${ }^{1}$ Food allergy with hen's egg generally are classified into type I allergic responses. Type I allergic responses are caused to the cross-linked structure between $\operatorname{IgE}$ on FceRI in mast and basophilic cells and the allergen. ${ }^{2}$ Then, these cells release various mediators such as histamine, cytokines, prostaglandins, and leukotrienes. These mediators induce the decrease of blood pressure and the drop of body temperature due to vascular relaxation, ${ }^{1,2}$ and the symptoms were called to anaphylactic shock. ${ }^{1}$ Moreover, the anamnestic history of anaphylactic shock affect the food allergy quality of life with the patients and their guardians. ${ }^{1,3,4}$ Therefore, it is expected that the amelioration of the severity of food allergy relieves 
the risk of anaphylactic shock and improves their quality of life.

Oral immunotherapy (OIT), which aims to induce desensitization by continuous intake of food allergens, is a new approach to food allergy treatment. ${ }^{1}$ However, Japanese pediatric guidelines do not recommend OIT because of the risk of inducing allergic symptoms during the treatment. ${ }^{1}$ Food materials such as raw egg, commercial spray-dried egg white (S-EW) powder, ${ }^{5}$ omelettes, ${ }^{6}$ and egg white (EW)containing cookies ${ }^{7}$ and muffins ${ }^{8}$ are used for OIT. However, the optimal OIT food has not yet been identified. Inuo reported that OIT involving the intake of partially hydrolyzed cow's milk protein-based formula safely improves tolerance to cow's milk in children with cow's milk allergy, compared to that with extensively hydrolyzed cow's milk protein-based formula intake. ${ }^{9}$ In high-risk infants, the hydrolyzed formula is safer than cow's milk formula. ${ }^{10}$ Thus, it is expected that peptides derived from allergens would be safe OIT food.

In an allergic mouse model, allergen-specific immunotherapy involving a hydrolyzed allergen or T-cell epitope peptides induced desensitization. ${ }^{11-15}$ The Kewpie Egg Corporation sells peptifine $^{\circ}(\mathrm{PF})$, an enzymatically hydrolyzed EW powder. The hydrogen sulfide odor of PF is considerably lower than that of other hydrolyzed EW peptide preparations, and the bitter flavor of PF is weaker than that of hydrolyzed peptides from soybean and casein. ${ }^{16}$ Generally, children are sensitive to bitter taste. Furthermore, some children with severe egg allergy feel nauseous after consuming cookies containing even trace amounts of egg. ${ }^{17}$ Therefore, the ability of OIT food to suppress the characteristic smell and taste of the allergen is critical. In the current study, we determined the ability of PF to induce immune tolerance and/or oral desensitization after 4-week intake using an EW allergic mouse model and compared the therapeutic effect of PF with that of commercial EW powder.

\section{Methods}

\section{Diets, EW allergic mouse model, and OIT protocol}

PF and S-EW were provided by the Kewpie Egg Corporation (Tokyo, Japan). Raw EW was diluted three times in water, mixed for $1 \mathrm{~h}$, filtered through a gauze, and freezedried (F-EW). All diets for the study were prepared in-house. All mice were fed $20 \%$ casein diet during allergic sensitization and after OIT for 4 weeks.

Care and treatment of the experimental animals were in accordance with the Mukogawa Women's University guidelines (Hyogo, Japan) for the ethical treatment of laboratory animals (no. FSN-01-2017-02-A). Female BALB/c mice $[\mathrm{n}=51$; 6 -week old; $21.5 \pm 0.1 \mathrm{~g}$ \{means \pm standard error (SE)\}] were purchased from Clea Japan (Tokyo, Japan). Mice were maintained under specific-pathogen-free conditions, and housed at $22^{\circ} \mathrm{C}$ under $60 \%$ humidity, and a 12-h light (08:00-20:00) and dark (20:00-08:00) cycle. For the EW allergic mouse model, animals were sensitized as described elsewhere. ${ }^{18}$ Forty-one mice were systemically sensitized to $100 \mu \mathrm{L}$ of $1 \mathrm{mg} / \mathrm{mL}$ EW solution and $100 \mu \mathrm{L}$ aluminum hydroxide gel (Imject $^{\text {ti }}$ Alum Adjuvant; Thermo Fisher Scientific Inc., Waltham, MA, USA) per mouse via intraperitoneal injections and triple oral allergen gavage (20 $\mathrm{mg} \mathrm{EW} /$ mouse). Ten mice received an intraperitoneal injection of $100 \mu \mathrm{L}$ saline and $100 \mu \mathrm{L}$ aluminum hydroxide gel per mouse (non-allergy group). The establishment of the EW allergic mouse model was confirmed by determining plasma levels of ovalbumin (OVA)-specific IgE at 1 week before OIT ( -1 week) and twice after an oral food challenge (OFC) before OIT. Forty-one EW allergic mouse model were divided into the experiment $(\mathrm{n}=30)$ for OIT using diets supplemented S-EW or PF and the experiment $(\mathrm{n}=11)$ for allergenicity of F-EW, S-EW and PF.

The OIT protocol was as described previously, ${ }^{18}$ with some modifications. Body weight and food intake were daily measured at 10:00 for day 1-28. Sensitized mice $(n=30$ total, with $\mathrm{n}=10$ per group) were housed individually in plastic cages and underwent 4 weeks of OIT with $1 \%$ PF diet (PF group) and 1\% S-EW diet (S-EW group); the nonOIT mice were fed $20 \%$ casein diet. The non-allergy group mice were fed $20 \%$ casein diet for 4 weeks. One mouse in the S-EW group died during the course of OIT, and a mouse in the non-OIT group died after intraperitoneal food challenge (IFC). Submandibular blood sampling ${ }^{19}$ was performed at the mid-point of OIT (2 weeks) and 1 day after OIT (4 weeks). OFC was performed at 3 days after OIT (4 weeks). IFC was conducted 4 days after OIT. Within 3 days of IFC, five mice from each group were sacrificed under isoflurane anesthesia to obtain the plasma and spleen at the study endpoint. Plasma samples were stored at $-40^{\circ} \mathrm{C}$ before analyses. At 4 days after IFC, vascular permeability was evaluated in the remaining animals (4-5 mice/group) according to published methods. ${ }^{1820}$

\section{Allergen challenge}

To quantitatively evaluate the severity of allergy in model mouse, we measured the change in the rectal temperature after OFC and IFC.

OFC was performed as described previously, ${ }^{18}$ with some modifications. Briefly, after an overnight fast, mice were orally challenged with $40 \mathrm{mg}$ F-EW in $400 \mu \mathrm{L}$ saline per mouse in the morning. Immediately before $\mathrm{OFC}$ and 15 min after OFC, rectal temperature was measured using a thermometer (KN-91-AD-1687-M; Natsume Seisakusyo Co. Ltd., Tokyo, Japan). IFC was performed as described, ${ }^{18,21}$ with some modifications. Thirty-nine mice were received an intraperitoneal injection of $0.2 \mathrm{mg}$ and $1 \mathrm{mg}$ F-EW in $200 \mu \mathrm{L}$ saline per mouse. Immediately before IFC, and $30 \mathrm{~min}$ and 60 min after IFC, rectal temperature was measured using a thermometer (as mentioned above).

Allergenicity of PF and S-EW were examined in the other mice sensitized with EW $(\mathrm{n}=11)$. Two days after OFC with F-EW, after an overnight fast, the mice were orally administered $40 \mathrm{mg} \mathrm{PF}$ and S-EW in $400 \mu \mathrm{L}$ saline per mouse in the morning. Immediately before and $15-90 \mathrm{~min}$ (at 15min intervals) after oral administration, rectal temperature was measured using a thermometer, as described above. 


\section{F-EW, S-EW and PF characteristics}

F-EW, S-EW and PF were analyzed using sodium dodecyl sulfate polyacrylamide-gel electrophoresis (SDS-PAGE) per the Laemmli method. ${ }^{22}$ Protein separations were performed on $5-20 \%$ gradient SDS-PAGE (ATTO Corporation, Tokyo, Japan). Disulfide bridges in samples were reduced by heating $\left(95^{\circ} \mathrm{C}, 2 \mathrm{~min}\right)$ in $1 \times$ SDS-PAGE sample buffer containing $2 \mathrm{w} / \mathrm{v} \%$ SDS, $5 \mathrm{v} / \mathrm{v} \% 2$-mercaptoethanol, and $10 \mathrm{v} / \mathrm{v} \%$ glycerol. After SDS-PAGE, proteins were detected by silver staining (Wako Pure Chemical Industries, Ltd. Osaka, Japan), following the manufacturer's instructions.

OVA and ovomucoid (OM) content in PF was determined using indirect enzyme-linked immunosorbent assay (ELISA). The diluted OVA (grade VI; Sigma-Aldrich, St. Louis, MO, USA) or OM, which was purified from EW by ethanol precipitation, standards and diluted PF solutions were coated in a 96-well flat bottom ELISA microplate (Nunc-Immuno Maxisorp, Thermo Fisher Scientific Inc) for overnight at $4^{\circ} \mathrm{C}$. After the blocking and washing, these standards and samples were detected by rabbit anti-OVA polyclonal antibodies (Sigma-Aldrich) or rabbit anti-OM polyclonal antibodies in our laboratory. The signal was detected using horseradish peroxidase (HRP)-conjugated goat anti-rabbit IgG polyclonal antibodies (Bethyl Laboratories, Montgomery, TX, USA). The HRP reactions were performed immediately in a final step of ELISA assay and the HRP substrate was used o-phenylenediamine. The reference curves were prepared using standard OVA and OM, and the concentrations of OVA and OM in PF solution were determined.

Assessment of plasma levels of OVA- and OM-specific antibodies, and cytokine secretion in spleen lymphocyte culture

Plasma levels of OVA- and OM-specific IgE and IgA were determined using capture ELISA. ${ }^{18}$ Mouse anti-OVA IgE monoclonal antibody E-G5 and mouse anti-OVA IgA monoclonal antibody 2G12E12 (Chondrex Inc., Redmond, WA, USA) were used as standards to determine the concentration of OVA-specific IgE and IgA. The levels of OM-specific IgE and IgA were presented as absorbance values at $492 \mathrm{~nm}$. The dilution of OM-specific IgE and IgA were 1:50. The HRP substrate used was o-phenylenediamine. The HRP reactions were performed immediately in a final step of ELISA assay and HRP reaction time points for OM-specific $\operatorname{IgE}$ at all three points were $20 \mathrm{mins}$, and for OM-specific IgA at 2 and 4 weeks, and at endpoint were $20 \mathrm{~min}$ and $11 \mathrm{~min}$, respectively.

Additionally, the levels of OVA- and OM-specific IgG1 and IgG2a were determined by indirect ELISA. ${ }^{18}$

Spleen lymphocytes were isolated from spleen homogenates using Lympholyte-M [Lympholyte(R)-M Cell Separation Media, Cedarlane Laboratories, ON, Canada]. Cells each mice were plated at a density of $1.5 \times 10^{7} /$ well in Roswell Park Memorial Institute (RPMI)-1640 complete medium in 24-well plates. The cells were stimulated with F-EW ( $2 \mathrm{mg}$ per well) and incubated at $37^{\circ} \mathrm{C}$ in the presence of $5 \% \mathrm{CO}_{2}$ for $48 \mathrm{~h}$. Concentrations of interleukin (IL) 4, IL-10, and interferon (IFN) $\gamma$ were assayed using ELISA (BioLegend, San Diego, CA, USA), according to the manufacturer's instructions.
Quantification of $\mathrm{CD}^{+} \mathrm{Foxp}^{+}$cells in spleen lymphocytes

To quantify the population of $\mathrm{CD} 4^{+}$Foxp $3^{+}$cells, $5.0 \times 10^{6}$ spleen lymphocytes per mouse isolated using Lympholyte-M (Cedarlane Laboratories) were transferred to a new tube. Extracellular staining was performed using fluorescein isothiocyanate (FITC)-conjugated anti-mouse anti-CD4 antibody (Clone; GK1.5, Biolegend), and intracellular staining was performed using Alexa 647-conjugated anti-mouse anti-Foxp3 antibody (Clone; 150D, BioLegend) according to the manufacturer's protocols. Cell signals were acquired using BD FACS Calibur and data were analyzed using Cell Quest Pro (BD Biosciences, Franklin Lakes, NJ, USA).

\section{Vascular permeability assessment}

The vascular permeability in dorsal skin can determine local allergic reactions. ${ }^{20}$ Mice received an intravenous injection of $200 \mu \mathrm{L}$ 1.5\% FITC-albumin (Sigma-Aldrich) per mouse under isoflurane anesthesia and intradermal injections of EW (at two positions; $20 \mu \mathrm{g} \mathrm{EW}$ in $50 \mu \mathrm{L}$ of Tyrode's solution per mouse) and Tyrode's solution (at two positions; $50 \mu \mathrm{L}$ per mouse) on the shaved dorsal skin. After $30 \mathrm{~min}$, the animals were sacrificed under isoflurane anesthesia to obtain the shaved dorsal skin and plasma samples. The fluorescent intensities of the dorsal skin and plasma extracts by formamide were measured using a fluorescent plate reader (Infinite M200; TECAN Ltd., Männedorf, Switzerland) with $485 \mathrm{~nm}$ excitation and $530 \mathrm{~nm}$ emission. The vascular permeability (50 $\mu \mathrm{L}$ plasma equivalent) was calculated as follows: EW, the mean of EW fluorescence value/plasma fluorescence value; Tyrode's, the mean of Tyrode's fluorescence value/plasma fluorescence value.

\section{Statistical analysis}

Values are presented as the means $\pm \mathrm{SE}^{23}$ Statistical differences, except for the vascular permeability and allergenicity of F-EW, S-EW and PF data, were analyzed using one-way analysis of variance (ANOVA), followed by Tukey's multiple comparison test. Vascular permeability values were compared using repeated two-way ANOVA, followed by Bonferroni's multiple comparison test. Statistical differences of vascular permeability were assessed using one-way ANOVA, followed by Tukey's multiple comparison test in all columns if repeated two-way ANOVA indicated a grouppoint interaction. Data regarding the allergenicity of F-EW, $\mathrm{S}$-EW and PF were analyzed using the paired $t$-test and Friedman's one-way ANOVA. P values less than 0.05 were considered as statistically significant. GraphPad Prism version 5.0 (GraphPad Software, San Diego, CA, USA) was used for all analyses.

\section{Results}

\section{Nutritional effect of diet supplementation with S-EW or PF}

Body weight at day 1 and day 28 , and body weight change for day 1-28 were not different among the all groups. However, the daily food intake was lower in the S-EW group than in the non-OIT and PF groups although not significant. 

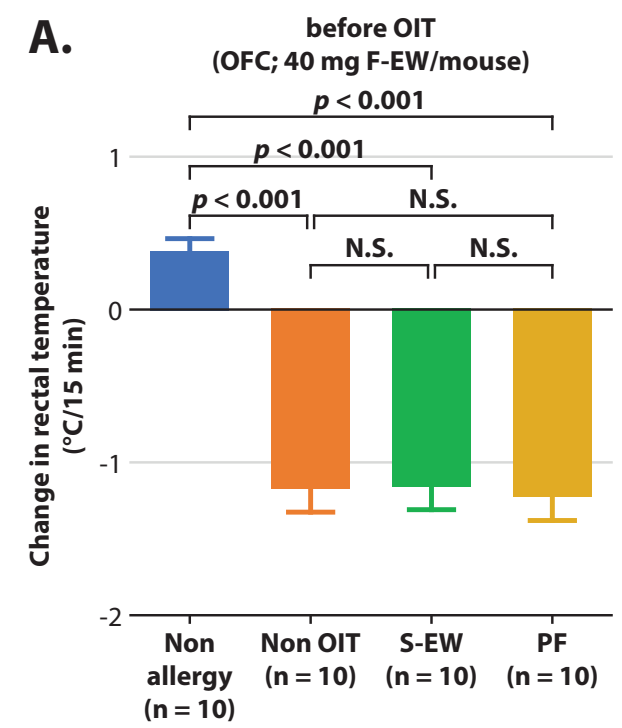

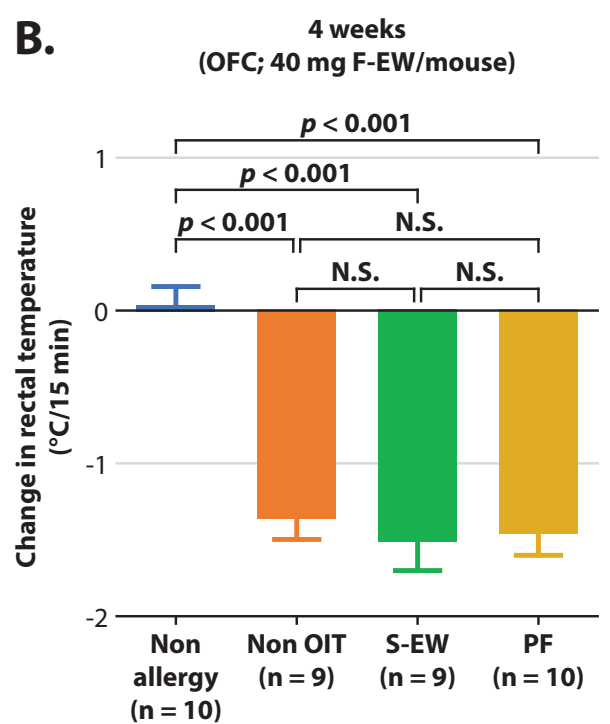

C.

IFC

(0.2 and $1 \mathrm{mg}$ F-EW/mouse)

D.

IFC (30 min after i.p. administration with $0.2 \mathrm{mg}$ F-EW/mouse) E. IFC (30 min after i.p. administration
with $1.0 \mathrm{mg}$ F-EW/mouse)
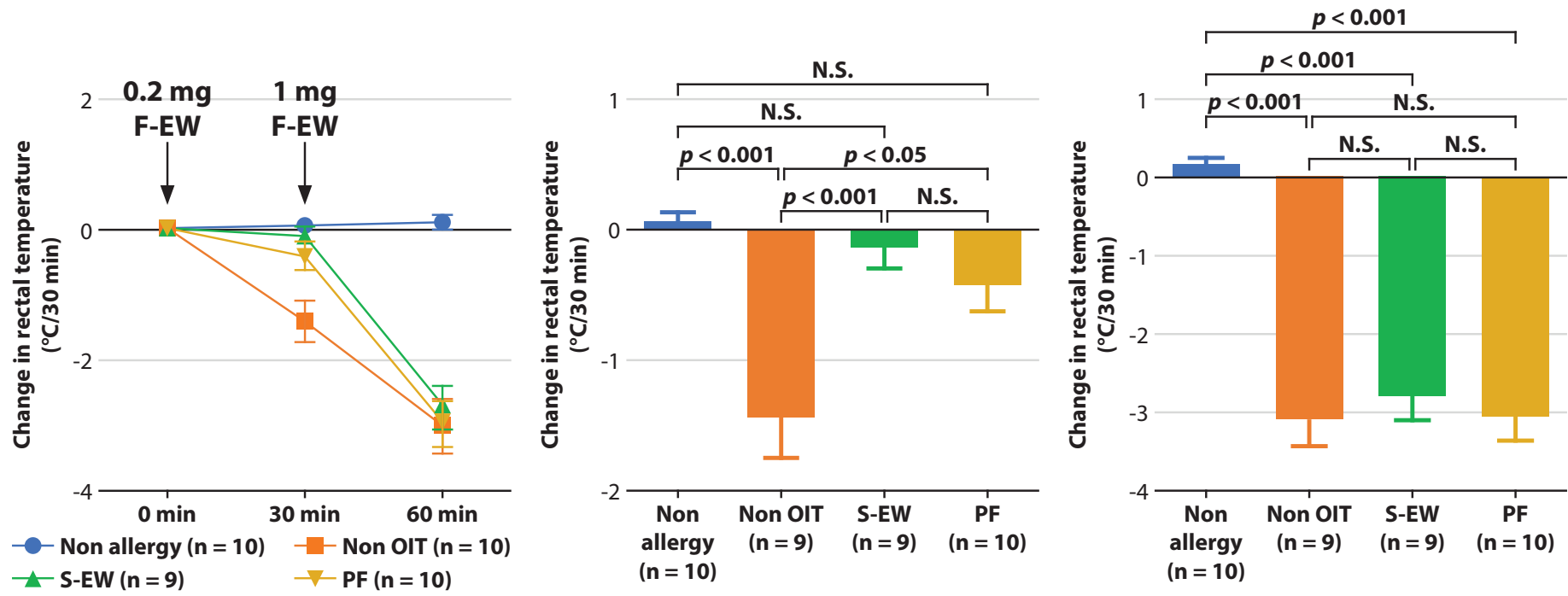

( $n=10$ )

Figure 1. Change in rectal temperature after OFC \{before OIT (A), 4 weeks (B)\} and IFC (C-E). Values show the mean \pm SE (n = 9-10). Statistical analysis (A, B, C and E) were performed using one-way ANOVA followed by Tukey's multiple comparison tests. $P$ values less than 0.05 were considered as statistically significant. N.S.: not significant

Outcome of OIT after diet supplementation with S-EW or PF

There were no differences in the change in rectal temperature after OFC in the allergy groups prior to OIT (Figure 1A). The change in rectal temperature in non-OIT, S-EW and PF groups after OFC at 4 weeks of OIT was comparable (Figure 1B).

After IFC with $0.2 \mathrm{mg}$ F-EW, the change in rectal temperature in the S-EW and PF groups was significantly suppressed than that in the non-OIT group (Figure $1 \mathrm{C}$ and 1D). In addition, after IFC with $1 \mathrm{mg}$ F-EW, the decrease in rectal temperature in non-OIT, S-EW and PF groups was comparable (Figure 1C and 1E). Furthermore, there was no significant difference in vascular permeability of skin injected with EW among the allergy groups (Figure 2).
Allergenicity of F-EW, S-EW and PF in vivo and SDS-PASE analysis

In other eleven mice with EW allergy, the rectal temperature after OFC with F-EW and S-EW was significantly dropped than that before the challenge (Figure $3 \mathrm{~A}$ and 3B). However, the rectal temperature after OFC with PF did not decrease compared to before the challenge (Figure 3B). Moreover, the change in rectal temperature after oral administration of PF significantly increased than that of F-EW and S-EW (Figure 3C).

SDS-PAGE analysis of the S-EW solution revealed multiple bands of various sizes, and the S-EW protein pattern was different from that of raw EW and F-EW. On the other hands, a faint $45 \mathrm{kDa}$ band in the PF lane was observed, and was consistent with the main band of the resolved OVA solution. The OM and OVA concentrations in $1 \mathrm{mg} / \mathrm{mL}$ solution of PF were $<0.2 \mu \mathrm{g} / \mathrm{mL}$ and $19.9 \mu \mathrm{g} / \mathrm{mL}$, respectively. 


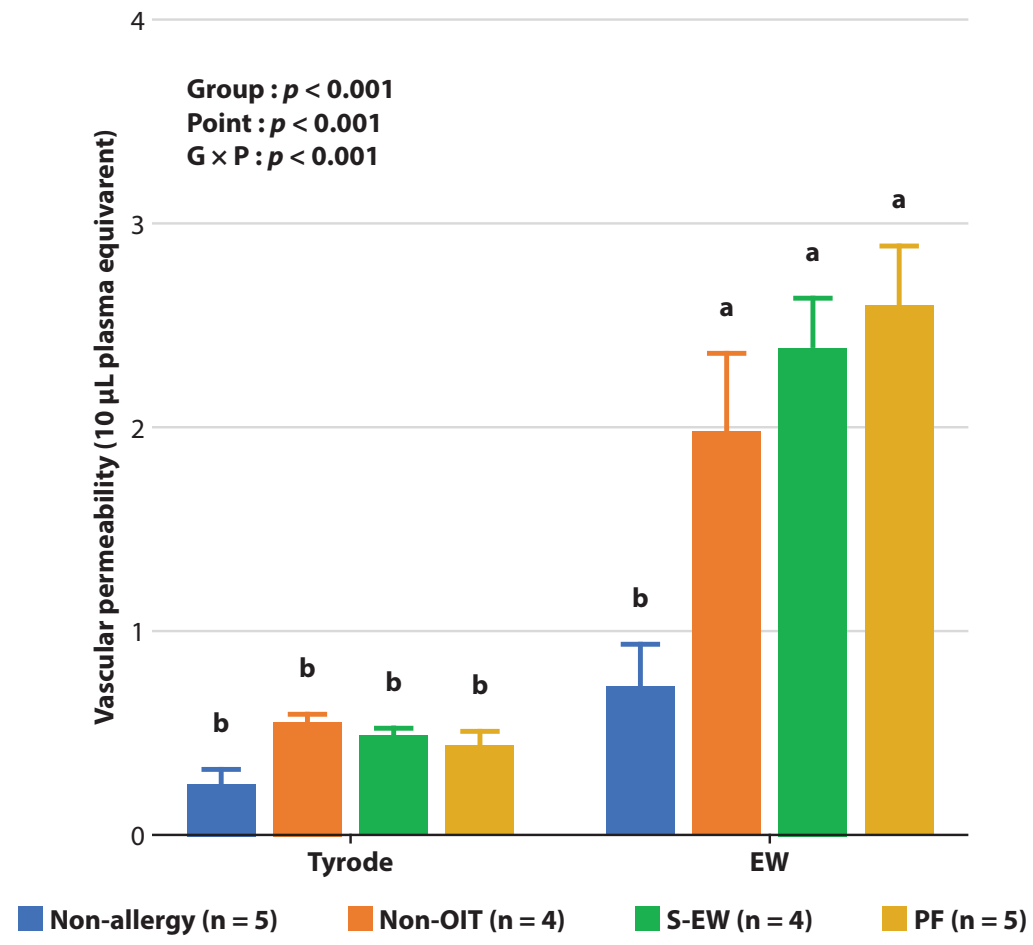

Figure 2. Vascular permeability. Values show the mean \pm SE $(n=4-5)$. Vascular permeability was calculated as follows: EW, the mean of EW fluorescence value/plasma fluorescence value; Tyrode's, the mean of Tyrode's fluorescence value/plasma fluorescence value. Statistical analysis were performed using one-way ANOVA followed by Tukey's multiple comparison tests for all columns when repeated two-way ANOVA indicated significant interactions. $P$ values less than 0.05 were considered as statistically significant. Significantly differences are indicated by different lower-case letters $(a, b)$, at $p<0.05$.

\section{A. Allergenicity of F-EW $(n=11)$} 39

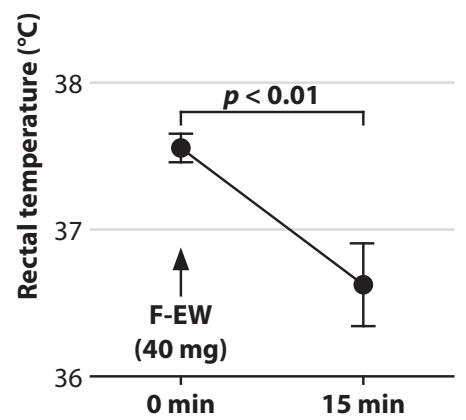

\section{B.} 39
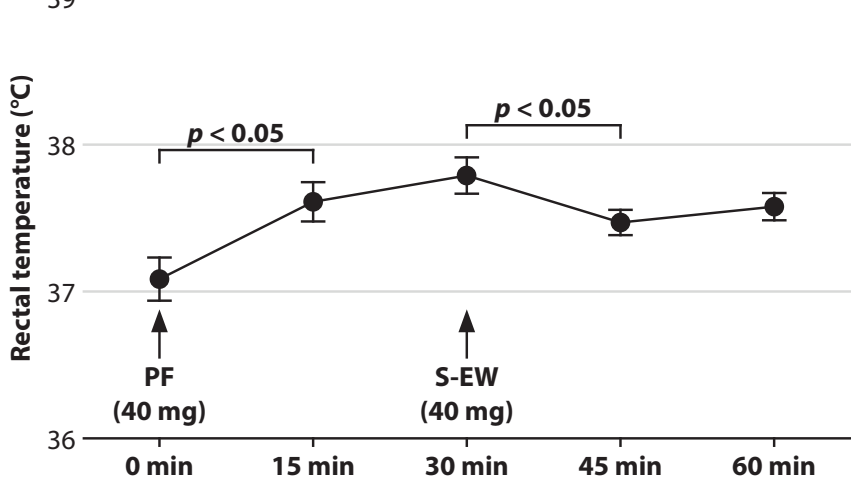
Allergenicity of PF and S-EW ( $n=11$ )
(OFC; $40 \mathrm{mg}$ PF and S-EW/mouse)
C. Allergenicity of F-EW, S-EW and C. PF (OFC; $\mathbf{4 0} \mathrm{mg}$ allergen/mouse)

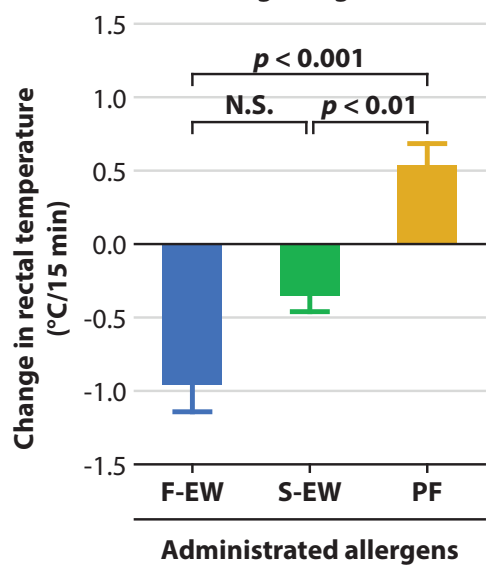

Figure 3. Allergenicity of F-EW, PF, and S-EW in vivo. The raw rectal temperatures $\{\mathrm{F}-\mathrm{EW}(\mathrm{A}), \mathrm{PF}$ and S-EW (B) $\}$ and the changes in rectal temperature after OFC with these allergens $(C)$ are presented as the mean \pm SE $(n=11)$. Statistical analysis (A and B) were performed using paired $t$-test (before vs. 15 min after oral administration) with each food. Statistical analysis of the change in rectal temperature after OFC with these allergens (C) was performed using Friedman's one-way ANOVA followed by the multiple comparison tests, which were used paired $t$-test and Bonferroni correction. $P$ values less than 0.05 were considered as statistically significant. N.S.: not significant 
Change in the plasma levels of OVA- and OM-specific antibodies in the EW allergic mouse fed S-EW or PF-containing diet

Plasma levels of OVA-specific IgE in the PF group at 2 weeks and 4 weeks of OIT were lower than those in the nonOIT and S-EW groups, although not significant (Table 1). However, at the study endpoint, they were comparable to those of the non-OIT, S-EW and PF groups (Table 1). Plasma levels of OM-specific IgE at 2 weeks of OIT were higher in the S-EW and PF groups than in the non-OIT group, although not significant (Table 2). However, at 4 weeks of OIT, plasma levels of OM-specific IgE in the S-EW and PF groups were lower than those in the non-OIT group, although not significant (Table 2). At the study endpoint, there were no differences in plasma levels of OM-specific IgE among the non-OIT, S-EW and PF groups (Table 2).
Plasma levels of OVA and OM-specific IgA in the PF group at 2 weeks and 4 weeks of OIT, and at the study endpoint were comparable to those of the non-OIT group (Table 1 and 2). However, the plasma levels of OVA-specific IgA in the S-EW group at 2 weeks and 4 weeks of OIT, and at the study endpoint were significantly higher (approximately 3-, 6-, and 2-fold, respectively) than those in the non-OIT group (Table 1). Furthermore, the plasma levels of OMspecific $\operatorname{IgA}$ in the S-EW group at 2 weeks and 4 weeks of OIT were significantly higher than those in the non-OIT and PF groups (Table 2). In contrast, at the study endpoint, the plasma levels of OM-specific IgA in the S-EW group were comparable with those in the non-OIT and PF groups (Table 2).

Table 1. Plasma concentrations of OVA-specific IgE and IgA at 2 weeks, 4 weeks, and study endpoint ${ }^{1}$

\begin{tabular}{|c|c|c|c|c|c|}
\hline & \multirow{2}{*}{ Non-allergy } & \multicolumn{3}{|c|}{ Allergy } & \multirow{2}{*}{$P$-value ${ }^{2}$} \\
\hline & & Non-OIT & S-EW & PF & \\
\hline \multicolumn{6}{|l|}{2 weeks } \\
\hline OVA-specific IgE $(\mu \mathrm{g} / \mathrm{mL})$ & $1.21 \pm 0.48^{\mathrm{b}}$ & $19.6 \pm 5.6^{\mathrm{a}}$ & $17.5 \pm 4.2^{\mathrm{a}}$ & $13.2 \pm 1.6^{\mathrm{ab}}$ & $<0.05$ \\
\hline OVA-specific IgA $(\mu \mathrm{g} / \mathrm{mL})$ & $0.411 \pm 0.123^{c}$ & $66.0 \pm 7.3^{b}$ & $187 \pm 19^{a}$ & $70.0 \pm 11.5^{\mathrm{b}}$ & $<0.001$ \\
\hline \multicolumn{6}{|l|}{4 weeks } \\
\hline OVA-specific IgE $(\mu \mathrm{g} / \mathrm{mL})$ & $0.796 \pm 0.321^{\mathrm{b}}$ & $24.7 \pm 5.7^{\mathrm{a}}$ & $22.7 \pm 7.2^{\mathrm{a}}$ & $18.5 \pm 1.5^{\mathrm{ab}}$ & $<0.01$ \\
\hline OVA-specific IgA $(\mu \mathrm{g} / \mathrm{mL})$ & $0.080 \pm 0.038^{\mathrm{b}}$ & $64.2 \pm 10.7^{\mathrm{b}}$ & $388 \pm 92^{\mathrm{a}}$ & $49.8 \pm 6.0^{\mathrm{b}}$ & $<0.001$ \\
\hline \multicolumn{6}{|l|}{ Endpoint } \\
\hline OVA-specific IgE $(\mu \mathrm{g} / \mathrm{mL})$ & $2.04 \pm 0.60^{\mathrm{b}}$ & $26.8 \pm 5.8^{\mathrm{a}}$ & $21.0 \pm 3.0^{\mathrm{a}}$ & $21.2 \pm 1.0^{\mathrm{a}}$ & $<0.001$ \\
\hline OVA-specific IgA $(\mu \mathrm{g} / \mathrm{mL})$ & $0.111 \pm 0.038^{c}$ & $61.1 \pm 12.7^{\mathrm{bc}}$ & $132 \pm 26^{\mathrm{a}}$ & $82.0 \pm 20.3^{\mathrm{ab}}$ & $<0.001$ \\
\hline
\end{tabular}

${ }^{1}$ Data are presented as mean \pm SE $(n=4-5)$.

${ }^{2}$ Statistical analysis were performed using one-way ANOVA, followed by Tukey's multiple comparison tests. $P$ values less than 0.05 were considered as statistically significant. Significantly differences are indicated by different lower-case letters (a, b, c), at $p<0.05$.

Table 2. Absorbance at $492 \mathrm{~nm}$ of plasma OM-specific IgE and IgA at 2 weeks, 4 weeks, and study endpoint ${ }^{1}$

\begin{tabular}{|c|c|c|c|c|c|c|}
\hline & \multirow{2}{*}{$\begin{array}{c}\text { HRP } \\
\text { Reaction } \\
\text { times }\end{array}$} & \multirow{2}{*}{ Non-allergy } & \multicolumn{3}{|c|}{ Allergy } & \multirow{2}{*}{$P$-value ${ }^{2}$} \\
\hline & & & Non-OIT & S-EW & PF & \\
\hline \multicolumn{7}{|l|}{2 weeks } \\
\hline OM-specific IgE & $20 \mathrm{~min}$ & $0.016 \pm 0.002^{\mathrm{b}}$ & $0.097 \pm 0.020^{\mathrm{ab}}$ & $0.163 \pm 0.040^{\mathrm{a}}$ & $0.128 \pm 0.018^{\mathrm{a}}$ & $<0.01$ \\
\hline OM-specific IgA & $20 \mathrm{~min}$ & $0.017 \pm 0.001^{\mathrm{c}}$ & $0.383 \pm 0.077^{\mathrm{b}}$ & $0.946 \pm 0.097^{\mathrm{a}}$ & $0.432 \pm 0.127^{\mathrm{b}}$ & $<0.001$ \\
\hline \multicolumn{7}{|l|}{4 weeks } \\
\hline OM-specific IgE & $20 \mathrm{~min}$ & $0.008 \pm 0.002^{\mathrm{b}}$ & $0.193 \pm 0.049^{\mathrm{a}}$ & $0.104 \pm 0.010^{\mathrm{ab}}$ & $0.115 \pm 0.017^{\mathrm{ab}}$ & $<0.01$ \\
\hline OM-specific IgA & $20 \mathrm{~min}$ & $0.008 \pm 0.001^{c}$ & $0.374 \pm 0.064^{\mathrm{b}}$ & $0.999 \pm 0.093^{\mathrm{a}}$ & $0.345 \pm 0.037^{\mathrm{b}}$ & $<0.001$ \\
\hline \multicolumn{7}{|l|}{ Endpoint } \\
\hline OM-specific IgE & $20 \mathrm{~min}$ & $0.014 \pm 0.004^{\mathrm{b}}$ & $0.354 \pm 0.073^{\mathrm{a}}$ & $0.312 \pm 0.067^{\mathrm{a}}$ & $0.344 \pm 0.080^{\mathrm{a}}$ & $<0.01$ \\
\hline OM-specific IgA & $11 \mathrm{~min}$ & $0.017 \pm 0.002^{\mathrm{b}}$ & $0.742 \pm 0.144^{\mathrm{a}}$ & $0.849 \pm 0.092^{\mathrm{a}}$ & $0.706 \pm 0.133^{\mathrm{a}}$ & $<0.001$ \\
\hline
\end{tabular}

${ }^{1}$ Data are presented as mean \pm SE $(n=4-5)$. The dilution rate was 1:50. The HRP substrate used was o-phenylenediamine.

${ }^{2}$ Statistical analysis were performed using one-way ANOVA, followed by Tukey's multiple comparison tests. $P$ values less than 0.05 were considered as statistically significant. Significantly differences are indicated by different lower-case letters (a, b, c), at $p<0.05$. 
Table 3. Concentrations of IFN- $\gamma$, IL-4, and IL-10 following in vitro stimulation of spleen lymphocytes with F-EW, and the percentage of $\mathrm{CD}^{+}$Foxp3 $^{+}$cells in spleen lymphocytes ${ }^{1}$

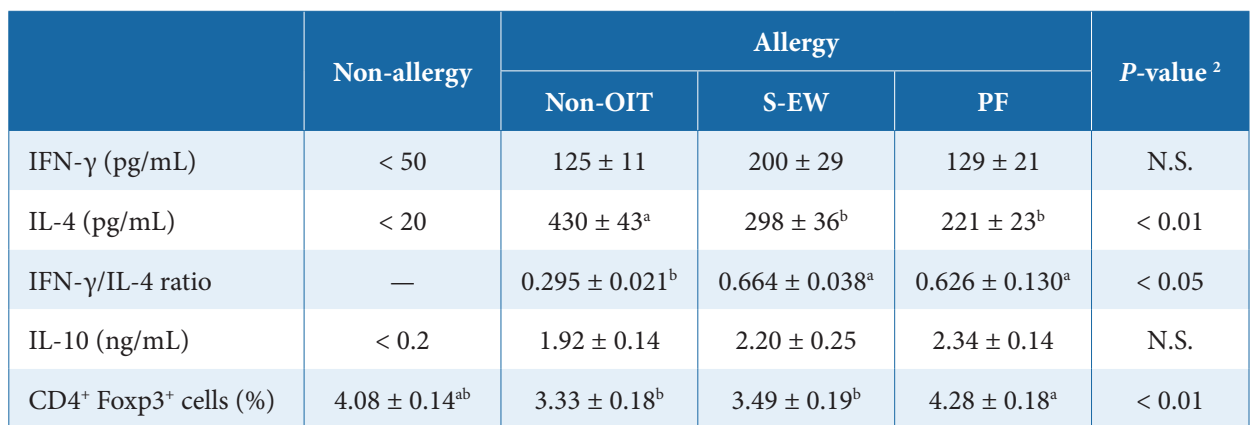

${ }^{1}$ Data are presented as mean \pm SE $(n=4-5)$. Cytokine secretions of splenocytes stimulated with 2 mg F-EW/well for 48 h were determined. The cytokine concentrations in the non-allergy group were below the limit of quantification in the manufacturers.

${ }^{2}$ Statistical analysis were performed using one-way ANOVA, followed by Tukey's multiple comparison tests. $P$ values less than 0.05 were considered as statistically significant. Significantly differences are indicated by different lower-case letters (a, b), at $p<0.05$.

N.S.: not significant

At the study endpoint, OVA-specific IgG1 in plasma lower in the S-EW and PF groups compared to the non-OIT group. On the other hands, at 2 weeks, the plasma levels of OMspecific IgG2a were higher than that of the non-OIT and PF groups.

Cytokine secretions and $\mathrm{CD}^{+}$Foxp $^{+}$cells in spleen lymphocytes of the EW allergic mouse model fed S-EW or PF-containing diet

In the spleen lymphocyte culture with EW, the concentration of IFN- $\gamma$, which is a cytokine secreted by helper T1 $\left(\mathrm{T}_{\mathrm{h}} 1\right)$ cells, ${ }^{2}$ was higher in the S-EW group than the non-OIT and PF groups although not significant (Table 3; $p=0.053$ ). The concentration of IL-4, which is a cytokine produced by helper T2 $\left(\mathrm{T}_{h} 2\right)$ cells, ${ }^{2}$ was significantly lower in the PF and S-EW groups than in the non-OIT group (Table 3 ). Furthermore, the IFN- $\gamma / \mathrm{IL}-4$ ratio, which reflects the $\mathrm{T}_{h} 1 /$ $\mathrm{T}_{\mathrm{h}} 2$ balance, was significantly higher in the S-EW and PF groups than in the non-OIT group (Table 3). There were no differences in the concentration of IL-10, which is an immune suppressor cytokine, ${ }^{2}$ between the allergy groups (Table 3 ). However, the percentage of $\mathrm{CD} 4^{+}$Foxp $3^{+}$splenocytes, which reflects the Treg population, in the PF group was significantly higher than that in the non-OIT and S-EW groups (Table 3).

\section{Discussion}

We investigated the ability of PF to induce immune tolerance and/or oral desensitization after 4-week intake in a mouse model of EW allergy and compared the therapeutic effect of PF with that of S-EW. Moreover, we measured the severity of food allergy by the change of rectal temperature after OFC and IFC because the various released allergic mediators induce the decrease of blood pressure and the drop of body temperature due to vascular relaxation. On the other hands, we did not use assessments by allergic symptom grades because the grades are subjectively observed and the grade judgement is difficult since a part of allergic symptom grades are indistinguishable to the behavers of mouse such as scratching and sleeping.
The mechanisms of oral tolerance are not completely clear. However, it is considered to relate the anergy (functional unresponsiveness), active immune suppression, and Treg mediated suppression. ${ }^{2}$ Therefore, we are thinking that a continuous allergens or peptides intake by OIT induces these phenomena. In an allergic mouse model, allergen-specific immunotherapy involving a hydrolyzed allergen or T-cell epitope peptides induced desensitization. ${ }^{11-15}$ In this study, after IFC with $0.2 \mathrm{mg}$ F-EW, the change in rectal temperature in the PF groups was significantly lower than that in the non-OIT group. Lozano-Ojalvo et al reported that the hydrolysated egg white protein modulated immune-cell responses. ${ }^{24,25}$ Moreover, they reported the hydrolysate of OVA with pepsin was more useful for oral tolerance and OIT than the intact OVA in an EW allergic mouse model. ${ }^{15}$ By contrast, a report showed that in a mouse model of EW allergy, the induced level of oral tolerance is comparable between raw egg and 5-fold hydrolyzed egg groups. ${ }^{26}$ Compared to those in the $1 \%$ F-EW group of our previous study, ${ }^{18}$ the desensitization in the $1 \%$ PF group was lower. It was reported that differences in the number and type of amino acids in the peptide affect the therapeutic effect by allergen-specific immunotherapy. ${ }^{11,12}$ Then, it needs to compare kinds of peptide in hydrolysated EW protein. On the other hands, a low allergenicity of hydrolysated proteins are consistent in almost studies. ${ }^{10,15,27}$ In this study, the allergenicity of PF was also lower than that of F-EW and S-EW in vivo. These results suggest that PF is safe as OIT food and that continuous intake of PF induced mild desensitization.

The vascular permeability in dorsal skin can determine local allergic reactions. ${ }^{20}$ The index relates the local histamine levels. However, in the experiments, there was no significant difference in vascular permeability among the non-OIT, S-EW and PF groups. Moreover, in our previous study, similar results were obtained..$^{18}$ Yamaki et al reported that histamineinduced serum exudation in the back skin increased from 0.31 to $1.25 \mathrm{mg}$ histamine/site in a dose-dependent manner and reached a plateau at $1.25-2.5 \mathrm{mg}$ histamine/site..$^{20}$ 
Therefore, we considered that the vascular permeability did not differ between the non-OIT, S-EW and PF groups because the therapeutic effects of OIT with S-EW or PF was not dramatic and was mild.

In this study, consumption of a diet supplemented with $1 \% \mathrm{~S}$-EW did not induce oral desensitization in a mouse model of EW allergy. This treatment effect in the S-EW group was weaker than that reported for $1 \%$ F-EW group in our previous study. ${ }^{18}$ Freeze-dry is a non-thermal drying method, and spray-dry is a thermal drying method. Handa and Kuroda showed that the incubation period required for sterilizing and improving the gelling properties of S-EW powder in the manufacture of S-EW promoted the Maillard reaction in the S-EW powder. ${ }^{28}$ Moreover, during the heating treatment in manufacture of S-EW, EW proteinglucose complexes were formed. ${ }^{28}$ In this SDS-analysis, the bands of S-EW was broad and high molecular compared with raw EW and F-EW. The heat treatment reduced IgE binding to OVA, and the Maillard reaction reduced $\operatorname{IgE}$ binding to OVA in experiments involving human serum. ${ }^{29}$ Furthermore, heated egg disrupts the IgE-reactive epitopes in OVA, and is susceptible to digestion. ${ }^{30}$ Hence, it appears that different drying methods alter the characteristics of the allergen and affect the therapeutic effects of OIT.

$\operatorname{IgE}$ is the most important antibody in allergic disease. ${ }^{1,2}$ The production of IgE and IgG1 is related to $\mathrm{T}_{\mathrm{h}} 2$ cells. $\mathrm{T}_{\mathrm{h}} 2$ cells release IL- 4 and IL-10, ${ }^{31}$ and IL- 4 promotes the production of IgE and IgG1 by B-cells. ${ }^{2}$ In this study, the plasma levels of OVA-specific IgE and IgG1 and OM-specific IgE were slightly lower in the PF group than in the non-OIT groups. Furthermore, IL-4 secretion from spleen lymphocytes treated with EW was significantly lower in the PF group than in the non-OIT group. Regulatory T-cells (Tregs) abundantly produce IL-10 but not IL-4. ${ }^{31}$ IL-10 inhibits the allergeninduced proliferative response of $\mathrm{T}_{\mathrm{h}} 2$ cells by IL- $4 .{ }^{32}$ Moreover, Treg cells control $\mathrm{T}_{\mathrm{h}} 2$ responses through the IRF4 interaction with Foxp $3 .^{33}$ There was no significant difference in the concentration of IL-10 in splenocyte cultures derived from the PF and non-OIT groups despite significant differences in the concentration of IL- 4 in the two groups. Thus, IL-10 in the PF group may have been released by Tregs. Furthermore, the percentage of $\mathrm{CD}^{+}$Foxp $^{+}$splenocytes in the $\mathrm{PF}$ group was significantly higher than that in the non-OIT groups. Therefore, we considered that continuous intake of $\mathrm{PF}$ promoted Treg differentiation. IFN- $\gamma$, a $\mathrm{T}_{\mathrm{h}} 1$ cytokine, selectively inhibits the proliferation of $\mathrm{T}_{\mathrm{h}} 2$ cells. ${ }^{32}$ In this experiments, IFN- $\gamma$ secretion from spleen lymphocytes treated with EW was higher in the S-EW group than in the non-OIT group. Moreover, the IL-4 secretion and the plasma levels of OVA- and OM-specific IgG1 in the S-EW group were lower than those of the non-OIT group. The IFN- $\gamma$ / IL-4 ratio was significantly higher in both the S-EW and $\mathrm{PF}$ groups than in the non-OIT group. Therefore, it was suggested that diet supplemented with $\mathrm{S}-\mathrm{EW}$ improved $\mathrm{T}_{\mathrm{h}} 1 /$ $\mathrm{T}_{\mathrm{h}} 2$ imbalance through the promotion of $\mathrm{T}_{\mathrm{h}} 1$ differentiation and the induction of IFN- $\gamma$ production. Furthermore, we considered that the mechanism of improvements of $T_{h} 1 / T_{h} 2$ imbalance differed between the S-EW and PF groups.
A correlation between desensitization and serum levels of allergen-specific IgA has been reported. ${ }^{7,34}$ However, in this study, we anticipated that a fed allergen might be promoting allergen-specific IgA production. We observed that plasma levels of OVA-specific IgA in the S-EW group were highest at 4 weeks of OIT, and the levels at the study endpoint rapidly decreased from the end of OIT. Furthermore, there were no differences in the plasma levels of OVA- and OM-specific IgA in the PF group compared to that in the non-OIT group at the three points. A report shows that serum allergen-specific IgA was not associated with natural or induced tolerance to egg white in children with egg allergy. ${ }^{35}$ We could not conclude whether the IgA increase was directly related to the OIT-mediated desensitization. Therefore, additional OIT experiments and studies are required before serum levels of allergen-specific IgA can be used as a biomarker of OIT outcome.

\section{Conclusions}

Our data indicated that diet supplemented with 1\% PF mildly ameliorated the severity of allergy in a mouse model of EW allergy, and that continuous intake of PF promoted Treg differentiation. PF might replace S-EW as OIT food for patients with egg allergy as it is safer than S-EW and the obtained desensitized effects by PF intake were comparable to that of S-EW. However, it needs more experiments or clinical trials to demonstrate the PF is better than S-EW because of not enough evidences of desensitization by intake of PF. The limitation in this study was that we did not prepare the group fed diet supplemented with 1\% F-EW as the OIT control. Future, it needs to examine the dose-response desensitization of OIT utilizing diet supplemented PF compared F-EW and to elucidate the detail molecular mechanism of desensitization by intake of PF.

\section{Acknowledgments}

We are grateful to Haruka Onishi, and Yu Nakamura for supporting this work. We thank Editage for English language editing.

\section{Conflicts of interest}

The authors have no conflict of interest to declare.

\section{Author contributions}

- $\mathrm{AM}$ and KT designed the study.

- AM, RK, and MM analyzed the samples.

- AM and KT drafted the manuscript. All authors approved the final manuscript.

\section{Funding}

This work was supported by JSPS KAKENHI (grant no. JP16K16293). PF and S-EW were provided by the Kewpie Egg Corporation. 


\section{References}

1. Ebisawa M, Ito K, Fujisawa T. Food Allergy Committee, Japanese Society of Pediatric Allergy and Clinical Immunology: Japanese pediatric guideline for food allergy 2016. Tokyo: Kyowa Kikaku Ltd. Press; 2016.

2. Abbas AK, Lichtman AH, Pillai S. Cellular and molecular immunology. $7^{\text {th }}$ ed. Philadelphia: Elsevier Saunders; 2011.

3. Cohen BL, Noone S, Muñoz-Furlong A, Sicherer SH. Development of a questionnaire to measure quality of life in families with a child with food allergy. J Allergy Clin Immunol. 2004;114(5):1159-63.

4. Mizuno Y, Ohya Y, Nagao M, DunnGalvin A, Fujisawa T. Validation and reliability of the Japanese version of the Food Allergy Quality of Life Questionnaire-Parent Form. Allergol Int. 2017;66(2):290-5

5. Burks AW, Jones SM, Wood RA, Fleischer DM, Sicherer SH, Lindblad RW, et al. Oral immunotherapy for treatment of egg allergy in children. N Engl J Med. 2012;367(3):233-43.

6. Sudo K, Taniuchi S, Takahashi M, Soejima K, Hatano Y, Nakano K, et al. Home-based oral immunotherapy (OIT) with an intermittent loading protocol in children unlikely to outgrow egg allergy. Allergy Asthma Clin Immunol. 2014;10(1):11.

7. Maeta A, Matsushima M, Muraki N, Asano M, Takaoka Y, Kameda M, et al. Low-dose oral immunotherapy using low-egg-allergen cookies for severe egg-allergic children reduces allergy severity and affects allergen-specific antibodies in serum. Int Arch Allergy Immunol. 2018;175(1-2):70-6.

8. Leonard SA, Sampson HA, Sicherer SH, Noone S, Moshier EL, Godbold J, et al. Dietary baked egg accelerates resolution of egg allergy in children. J Allergy Clin Immunol. 2012;130(2):473-80.

9. Inuo C, Tanaka K, Suzuki S, Nakajima Y, Yamawaki K, Tsuge I, et al. Oral immunotherapy using partially hydrolyzed formula for cow's milk protein allergy: A randomized, controlled trial. Int Arch Allergy Immunol. 2018;177(3):259-68.

10. Vandenplas Y, Bhatia J, Shamir R, Agostoni C, Turck D, Staiano A, et al. Hydrolyzed formulas for allergy prevention. J Pediatr Gastroenterol Nutr. 2014;58(5):549-52.

11. Yang M, Yang C, Mine Y. Multiple T cell epitope peptides suppress allergic responses in an egg allergy mouse model by the elicitation of forkhead box transcription factor 3- and transforming growth factor-beta-associated mechanisms. Clin Exp Allergy. 2010;40(4):668-78.

12. Rupa $\mathrm{P}$, Mine Y. Oral immunotherapy with immunodominant T-cell epitope peptides alleviates allergic reactions in a $\mathrm{Balb} / \mathrm{c}$ mouse model of egg allergy. Allergy. 2012;67(1):74-82.

13. Kulis M, Macqueen I, Li Y, Guo R, Zhong XP, Burks AW. Pepsinized cashew proteins are hypoallergenic and immunogenic and provide effective immunotherapy in mice with cashew allergy. J Allergy Clin Immunol. 2012;130(3):716-23.

14. Yang M, Yang C, Nau F, Pasco M, Juneja LR, Okubo T, et al Immunomodulatory effects of egg white enzymatic hydrolysates containing immunodominant epitopes in a BALB/c mouse model of egg allergy. J Agric Food Chem. 2009;57(6):2241-8.

15. Lozano-Ojalvo D, Pérez-Rodríguez L, Pablos-Tanarro A, Molina E, López-Fandiño R. Hydrolysed ovalbumin offers more effective preventive and therapeutic protection against egg allergy than the intact protein. Clin Exp Allergy. 2017;47(10):1342-54.

16. Watanabe K. [Function of egg white peptide "Peptifine"]. Jpn Food Sci. 2013;52(1):17-24. Japanese.

17. Maeta A, Muraki N, Ishibe E, Mori H, Omuro K, Takaoka Y, et al. [Survey on intake status of low-allergy egg bolo in children with egg allergy]. J Jpn Soc Pediatr Clin Allergy. 2018:16(3);354-62. Japanese.
18. Maeta A, Matsushima M, Katahira R, Sakamoto N, Takahashi K. Diets supplemented with $1 \%$ egg white induce oral desensitization and immune-tolerance in egg white specific allergic mouse model. Int Arch Allergy Immunol. 2018;176(3-4):205-14.

19. Golde WT, Gollobin P, Rodriguez LL. A rapid, simple, and humane method for submandibular bleeding of mice using a lancet. Lab Anim. 2005;34(9):39-43.

20. Yamaki K, Takano-Ishikawa $\mathrm{Y}$, Goto $\mathrm{M}$, Kobori $\mathrm{M}$, Tsushida T. An improved method for measuring vascular permeability in rat and mouse skin. J Pharmacol Toxicol Methods. 2002;48(2):81-6.

21. Leonard SA, Martos G, Wang W, Nowak-Węgrzyn, A, Berin MC. Oral immunotherapy induces local protective mechanisms in the gastrointestinal mucosa. J Allergy Clin Immunol. 2012;129(6):1579-87.

22. Laemmli UK. Cleavage of structural proteins during the assembly of the head of bacteriophage T4. Nature. 1970;227(5259):680-5.

23. Cumming G, Fidler F, Vaux DL. Error bars in experimental biology. J Cell Biol. 2007;177(1):7-11.

24. Lozano-Ojalvo D, Molina E, López-Fandiño R. Hypoallergenic hydrolysates of egg white proteins modulate allergen responses induced ex vivo on spleen cells from sensitized mice. Food Res Int. 2016; 89(Pt 1):661-9.

25. Lozano-Ojalvo D, Molina E, López-Fandiño R. Hydrolysates of egg white proteins modulate $\mathrm{T}$ - and $\mathrm{B}$-cell responses in mitogen-stimulated murine cells. Food Funct. 2016;7(2):1048-56.

26. Hacini-Rachinel F, Vissers YM, Doucet-Ladevéze R, Blanchard C, Demont A, Perrot M, et al. Low-allergenic hydrolyzed egg induces oral tolerance in mice. Int Arch Allergy Immunol. 2014;164(1):64-73.

27. Ueno HM, Kato T, Ohnishi H, Kawamoto N, Kato Z, Kaneko H, et al. T-cell epitope-containing hypoallergenic $\beta$-lactoglobulin for oral immunotherapy in milk allergy. Pediatr Allergy Immunol. 2016;27(8): 818-24.

28. Handa A, Kuroda N. Functional improvements in dried egg white through the Maillard reaction. J Agric Food Chem. 1999;47(5):1845-50.

29. Jiménez-Saiz R, Belloque J, Molina E, López-Fandiño R. Human immunoglobulin E (IgE) binding to heated and glycated ovalbumin and ovomucoid before and after in vitro digestion. J Agric Food Chem. 2011;59(18):10044-51.

30. Watanabe H, Toda M, Sekido H, Wellner A, Fujii T, Henle T, et al. Heat treatment of egg white controls allergic symptoms and induces oral tolerance to ovalbumin in a murine model of food allergy. Mol Nutr Food Res. 2014;58(2):394-404.

31. Groux H, O'Garra A, Bigler M, Rouleau M, Antonenko S, de Vries JE, et al. A CD4+ T-cell subset inhibits antigen-specific T-cell responses and prevents colitis. Nature. 1997;389(6652): 737-42.

32. Del Prete G, De Carli M, Almerigogna F, Giudizi MG, Biagiotti R, Romagnani S. Human IL-10 is produced by both type 1 helper (Th1) and type 2 helper (Th2) T cell clones and inhibits their antigen-specific proliferation and cytokine production. J Immunol. 1993;150(2):353-60.

33. Zheng Y, Chaudhry A, Kas A, deRoos P, Kim JM, Chu TT, et al. Regulatory T-cell suppressor program co-opts transcription factor IRF4 to control $\mathrm{T}(\mathrm{H}) 2$ responses. Nature. 2009;458(7236):351-6.

34. Wright BL, Kulis M, Orgel KA, Burks AW, Dawson P, Henning AK, et al. Component-resolved analysis of $\operatorname{IgA}$, IgE, and IgG4 during egg OIT identifies markers associated with sustained unresponsiveness. Allergy. 2016;71(11):1552-60.

35. Vazquez-Ortiz M, Pascal M, Juan M, Alsina L, Martín-Mateos MA, Plaza AM. Serum allergen-specific IgA is not associated with natural or induced tolerance to egg in children. Allergy. 2013;68(10):1327-32. 